\title{
Extreme Light Concentration Inside Plasmonic Gold and Silver Nanoparticles During Laser Irradiation
}

\author{
L. G. Astafyeva ${ }^{1}$ V. K. Pustovalov ${ }^{2}$
}

Received: 19 December 2014/Accepted: 24 April 2015/Published online: 7 May 2015

(C) Springer Science+Business Media New York 2015

\begin{abstract}
Computer modeling of the distributions of laser radiation intensities concentrated inside spherical gold and silver nanoparticles with the radii in the range $10-100 \mathrm{~nm}$ was carried out during laser irradiation with wavelengths 400,532 , and $800 \mathrm{~nm}$. Distributions of laser intensity are inhomogeneous for investigated values of nanoparticle sizes and laser wavelengths. Here, we show new effect of extreme light intensity concentration in shadow hemisphere of gold and silver nanoparticles with localizing light at the nanoscale for selected values of nanoparticle sizes and radiation wavelengths. No doubt that this effect exists for different NPs and wavelengths. These results can be applied in nanophotonics for construction of new plasmonic antennas and photonic components, and for the laser technologies of the preparation and the treatment of NPs with definite sizes, shapes, structure, size distribution, etc.
\end{abstract}

Keywords Gold nanoparticles · Silvernanoparticles · Internal intensity distribution $\cdot$ Laser irradiation

\section{Introduction}

Recently, many research efforts have been focused on the investigations of metal nanoparticles because of their unique size-dependent physical and chemical properties. Metal

L. G. Astafyeva

astafev@dragon.bas-net.by

1 B.I. Stepanov Institute of Physics, National Academy of Sciences of Belarus, Pr. Independency, 68, Minsk 220072, Belarus

2 Belarusian National Technical University, Pr. Independency, 65, Minsk 220013, Belarus nanoparticles (NPs) are used in many different fields of research and technology including nanophotonics and nanoelectronics, catalysis, novel materials, and nonlinear devices. In particular, gold and silver NPs have been studied aiming at a variety of medical applications, such as laser biomedicine and cancer research, biosensors.

The emerging fields of nanophotonics and plasmonics are yielding the methods for guiding and localizing light at the nanoscale, well below the scale of the light wavelength. Important interest in the investigation of optical (laser) radiation interaction with nanostructures and NPs is focused on the possibility of the radiation intensity concentration near plasmonic nanostructures during irradiation. Many publications deal with intensity concentration for different applications in nanophotonics and nanoelectronics [1-11]. The ability of plasmonic nanostructures to concentrate light into deepsubwavelength volumes and to manipulate light well below the classical diffraction limit has promoted their use in nanophotonics. Plasmonic nanostructures, antennas, and light concentrators can change light-matter interactions at a fundamental level and also convert optical radiation into intense localized distributions. The integration of existing microand nanoelectronic devices with plasmonic structures has been realized for near-field scanning optical and laser microscopy, subwavelength optical imaging, new nanoscale photonic components, photodetectors, etc.

On the other hand, it is very important to prepare metal NPs with definite sizes, shapes, and structure for different applications in nanotechnology. Several attempts have been made with metal NPs in a solution by means of lasers having various performances [12-20]. These attempts have been made to fabricate nanoscale NPs of scientific and industrial interest in a size- and geometry-controlled manner. A laser-induced preparations and treatments of metal NPs and nanostructures is one of the most promising methods. It is necessary to 
elucidate the mechanism of how the preparation proceeds to establish these methods.

It is principally interesting to carry out the investigation of optical (laser) radiation interaction with nanostructures and NPs focused on the possibility of radiation intensity concentration inside plasmonic metallic NPs during irradiation. Investigation of radiation intensity distributions inside and outside dielectric microparticles and aerosol particles illuminated by an electromagnetic wave was carried out [21-23]. The distributions of laser intensity inside NPs will determine the mechanisms and results of laser-NP interaction. But there is lacking data, and their analysis of the radiation intensity concentration inside plasmonic NPs at all.

Computer modeling of the distributions of laser radiation intensities inside spherical gold and silver NPs with the radii in the range 5-100 $\mathrm{nm}$ during laser irradiation with the wavelengths 400,532 , and $800 \mathrm{~nm}$ have been carried out in this paper. New effect of extreme light intensity concentration in shadow hemisphere of gold and silver NPs for selected values of NP sizes and radiation wavelengths was established. These results can be applied in nanophotonics for construction of new plasmonic antennas and photonic components, and for the laser processing of NPs for scientific and industrial purposes.

\section{Results of Computer Modeling of Internal Laser Intensity Distributions Inside Gold and Silver NPs and Discussions}

We consider the interaction of plane electromagnetic wave of laser (optical) radiation with spherical gold and silver NPs on the base of computer modeling for the purpose of the investigation of the laser radiation intensity distributions inside spherical NPs. NPs are placed in water medium with refraction index $n_{\lambda}=$ $1.339(\lambda=400 \mathrm{~nm}), 1.3337(\lambda=532 \mathrm{~nm})$, and 1.329 $(\lambda=800 \mathrm{~nm})$ [24]. We have to note that for surrounding media with refraction indexes in the range $n_{\lambda}=1.2-1.55$ (silica, some dielectrics, etc.) presented below, results can be also applied. Optical properties of gold and silver were taken from [25]. The radiation intensity was normalized on the intensity of initial wave $I_{0}$ at irradiated surface of NP and normalized intensity is equal $I_{\mathrm{n}}=I / I_{0}$ (see Figs. 1 and 2) and was determined in arbitrary units. Method of computer modeling of internal intensity distributions inside nanoparticles is presented in $[22,23,26]$.

The processes of the radiation interaction with spherical NPs are simultaneously taking part in the formation of the intensity distributions inside NPs. They include combined action of the absorption, scattering, extinction of radiation by $\mathrm{NP}$, and diffraction of radiation on spherical NP. The results are determined by NP radius, optical indexes of the NP material (metal), and refraction index of surrounding nonabsorbing medium that are corresponding to the selected wavelength.

Figure 1a-f presents the distributions of the normalized radiation intensity $I_{\mathrm{n}}$ along the main diameter, coinciding with the propagation direction of the incident irradiation, inside spherical gold (Au) NPs with the radii $r_{0}=10,20,40,60,80$, and $100 \mathrm{~nm}$ (Fig. $1 \mathrm{a}-\mathrm{c}$ ) and dependences of the efficiency factors of absorption $K_{\mathrm{abs}}$, scattering $K_{\mathrm{sca}}$, and extinction $K_{\mathrm{ext}}$ on NP radii $r_{0}$ in the range $5-100 \mathrm{~nm}$ (Fig. 1d-f) for the radiation wavelengths $\lambda=400 \mathrm{~nm}$ (Fig. $1 \mathrm{a}, \mathrm{d}$ ), $532 \mathrm{~nm}$ (Fig. 1b, e), $800 \mathrm{~nm}$ (Fig. 1c, f). Efficiency factors of absorption $K_{\mathrm{abs}}$, scattering $K_{\mathrm{sca}}$, extinction, and $K_{\mathrm{ext}}$ are determined in [2] as absorption, scattering, and extinction cross sections divided by the cross sectional area of NP.

The intensity $I_{n}$ decreases linearly along NP main diameter for the wavelength $400 \mathrm{~nm}$ from irradiated surface to shadow surface for small $\mathrm{Au}$ NPs with the radii $r_{0}=10$ and $20 \mathrm{~nm}$ because of extinction of radiation inside NP. Intensity decreases from irradiated surface to central part of NP, and it has minimal value here for $r_{0}>40 \mathrm{~nm}$. The intensity $I_{\mathrm{n}}$ increases after that in the NP shadow hemisphere. The efficiency factor of extinction $K_{\text {ext }}$ increases up to the maximal value $K_{\text {ext }}^{\max } \sim 3.12$ at $r_{0}=48 \mathrm{~nm}$ and approximately constant with increasing of $r_{0}$. The values of $K_{\text {abs }}$ are bigger than $K_{\mathrm{sca}}$ for the range of $100 \mathrm{~nm}>r_{0}>10 \mathrm{~nm}$.

Another situation exists for Au NPs under action of laser wavelength $\lambda=532 \mathrm{~nm}$ closing to plasmon resonance wavelength for these NPs. The relative intensity $I_{\mathrm{n}}$ slightly decreases along main NP diameter from irradiated surface to shadow surface for small radiuses $r_{0}=$ 10 and $20 \mathrm{~nm}$. But for $r_{0} \geq 40 \mathrm{~nm}$ relative intensity $I_{\mathrm{n}}$ decreases from irradiated surface to center of NP and $I_{\mathrm{n}}$ has minimal value at the central part of NP. The intensity $I_{\mathrm{n}}$ increases after that in shadow hemisphere and achieves up to relative values of about $\sim 1.3$ that are higher than $I_{n}$ at irradiated surface. We have to note especially $I_{\mathrm{n}}$ distribution for $r_{0}=60 \mathrm{~nm}$ because in this case the value of $I_{\mathrm{n}}$ has substantial maximum $I_{\mathrm{n}}\left(r / r_{0}=\right.$ $1) \approx 3.8$, and it is greater than the values of $I_{\mathrm{n}}\left(r / r_{0}=I\right)$ for other $r_{0}$. The maximum values of the relative intensity $I_{n}$ are placed at the shadow particle surface with nonmonotonic dependence of $I_{\mathrm{n}}\left(r / r_{0}=1\right)$ on increasing value of $\mathbf{r}_{0}$. This is unexpected result for $\lambda=532 \mathrm{~nm}$ in comparison with $\lambda=400 \mathrm{~nm}$.

Efficiency factor of extinction $K_{\text {ext }}$ increases up to the maximal value $\sim 5.6$ at $r_{0}=40 \mathrm{~nm}$, after that $K_{\text {ext }}$ has light minimum at $r_{0}=60 \div 70 \mathrm{~nm}$ and slightly increases with increasing of $r_{0}$. The values of $K_{\text {abs }}$ are bigger than $K_{\text {sca }}$ for the range of $52 \mathrm{~nm}>r_{0}>10 \mathrm{~nm}$. We have to note that the maximal value of $I_{\mathrm{n}}$ (at $r_{0}=60 \mathrm{~nm}$ ) is correlated with minimal value of $K_{\text {ext }}$ (at $r_{0}=60 \mathrm{~nm}$ ). It means that inhomogeneous distributions of internal relative intensity $I_{n}$ 
Fig. 1 Distributions of the relative radiation intensity $I_{\mathrm{n}}$ along the main diameter of NP inside gold (Au) NPs with the radii $r_{0}=10(1), 20(2), 40(3), 60$ (4), 80 (5), $100(6) \mathrm{nm}(\mathbf{a}, \mathbf{b}, \mathbf{c})$ and dependences of $K_{\text {abs }}$ (solid), $K_{\text {sca }}\left(\right.$ dashed), and $K_{\text {ext }}$ (dasheddotted $)$ on NP radii $r_{0}(\mathbf{d}, \mathbf{e}, \mathbf{f})$ for the wavelengths $\lambda=400 \mathrm{~nm}$ (a, d), $532 \mathrm{~nm}(\mathbf{b}, \mathbf{e})$, and $800 \mathrm{~nm}(\mathbf{c}$, f). The direction of laser radiation propagation is from left to right side. Vertical lines 1-6 (d, e, f) locate the values of $K_{\mathrm{abs}}, K_{\mathrm{sca}}$, and $K_{\text {ext }}$ for selected values of $r_{0} 1-6$ $(\mathbf{a}, \mathbf{b}, \mathbf{c})$ a

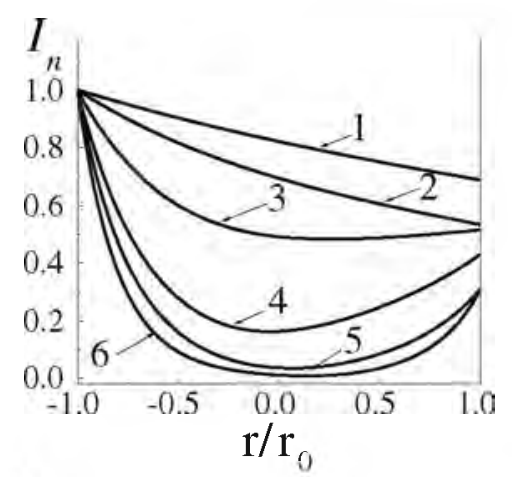

b

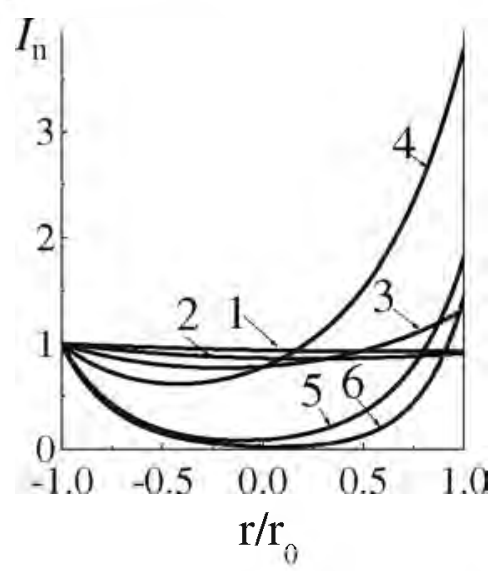

C

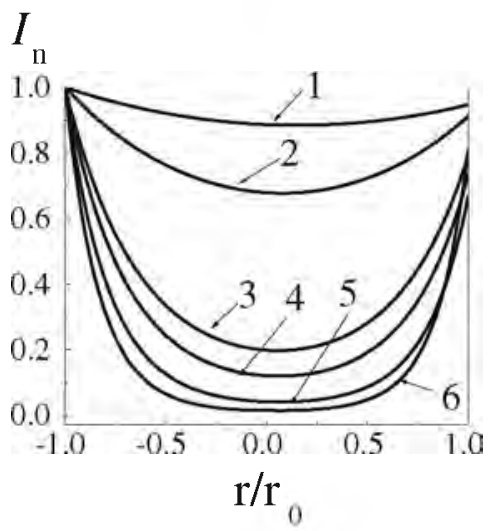

d

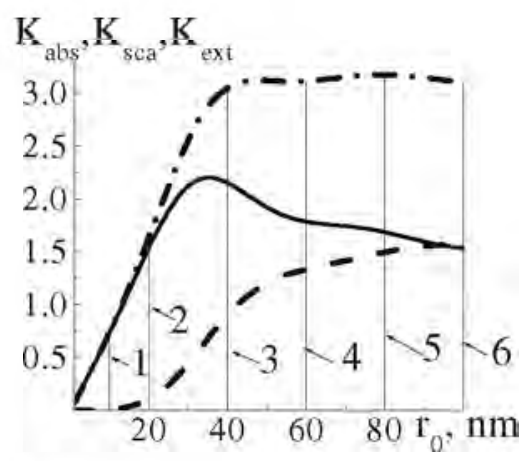

e

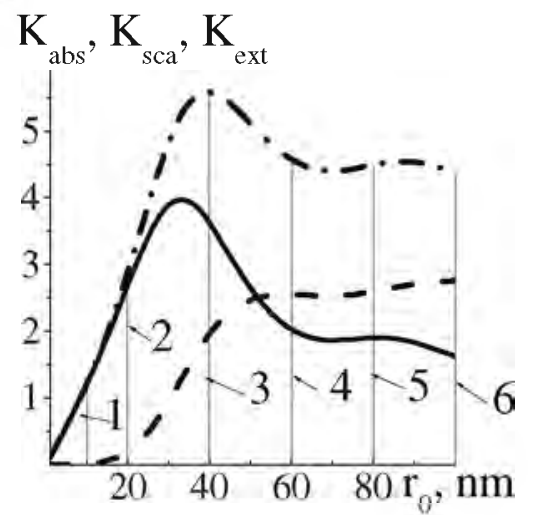

f

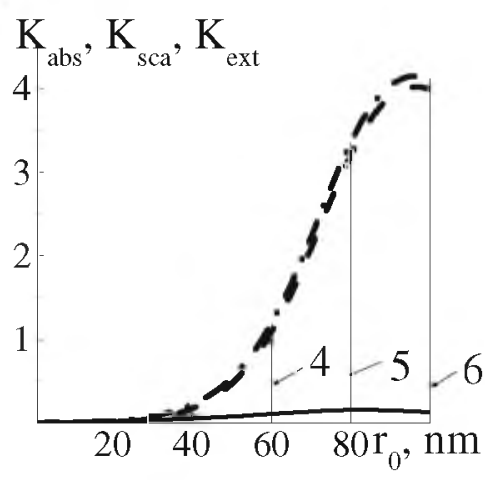

near shadow nanoparticle surfaces are determined by plasmon resonance dependence of $K_{\text {ext }}$ on $r_{0}$.

The relative intensity $I_{\mathrm{n}}$ decreases from irradiated surface to center of NP for the wavelength $\lambda=800 \mathrm{~nm}$. The intensity $I_{\mathrm{n}}$ has minimal value at the central part of NP, and it increases after that up to relative value of $I_{\mathrm{n}} \sim 0.7-0.8$. The character of the intensity distributions inside the gold NPs are inhomogeneous with the maximum value of the intensity on the illuminated surface of the NPs. The distributions of $I_{\mathrm{n}}\left(r / r_{0}\right)$ have practically symmetric visible pictures relatively to the NP center $r / r_{0}=0$. In this case $K_{\text {ext }}$ and $K_{\text {sca }}$ monotonously increase, and they are much bigger than $K_{\mathrm{abs}}$ and they have no minimal values in the range of $r_{0}=10-100 \mathrm{~nm}$.

Figure 2a-f presents the distributions of normalized radiation relative intensity $I_{\mathrm{n}}$ along the main diameter, coinciding with the propagation direction of the incident irradiation, inside spherical silver (Ag) NPs with the radii $r_{0}=10,20,40,60$, 80 , and $100 \mathrm{~nm}$ of NP (Fig. 2a-c) and dependences of $K_{\mathrm{abs}}$, $K_{\text {sca }}$ and $K_{\text {ext }}$ on NP radii $r_{0}$ in the range $r_{0}=10-100 \mathrm{~nm}$ (Fig. $2 \mathrm{~d}-\mathrm{f}$ ) for the radiation wavelengths $\lambda=400 \mathrm{~nm}$ (Fig. 2a, d), $532 \mathrm{~nm}$ (Fig. 2b, e), $800 \mathrm{~nm}$ (Fig. 2c, f). A11 designations are the same ones as in Fig. 1. 
Fig. 2 Distributions of the

relative radiation intensity $I_{\mathrm{n}}$ along the main diameter of NP inside silver $(\mathrm{Ag}) \mathrm{NPs}$ with radii $r_{0}=10(1), 20(2), 40(3), 60(4)$, $80(5), 100(6) \mathrm{nm}(\mathbf{a}, \mathbf{b}, \mathbf{c})$ and dependences of $K_{\text {abs }}($ solid $) K_{\text {sca }}$ (dashed), $K_{\text {ext }}$ (dashed-dotted) on $\mathrm{NP}$ radii $r_{0}(\mathbf{d}, \mathbf{e}, \mathbf{f})$ for $\lambda=400 \mathrm{~nm}$ (a, d), $532 \mathrm{~nm}(\mathbf{b}, \mathbf{e})$, and $800 \mathrm{~nm}$ $(\mathbf{c}, \mathbf{f})$. The direction of laser radiation propagation is from left to right side. Vertical lines 1-6 (d, e, f) locate the values of $K_{a b s,}$,

$K_{s c a}$, and $K_{\text {ext }}$ for selected values of $r_{0} 1-6(\mathbf{a}, \mathbf{b}, \mathbf{c})$ a

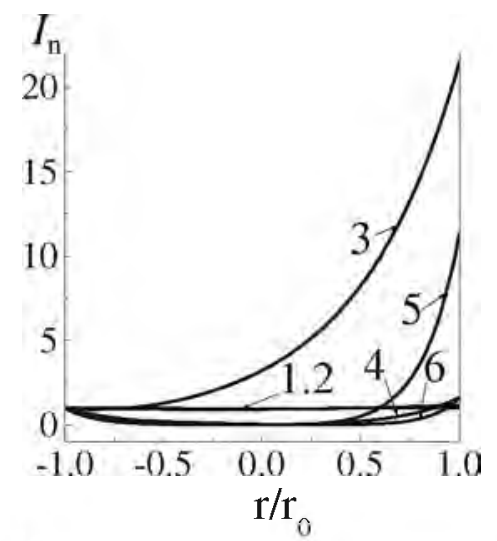

b

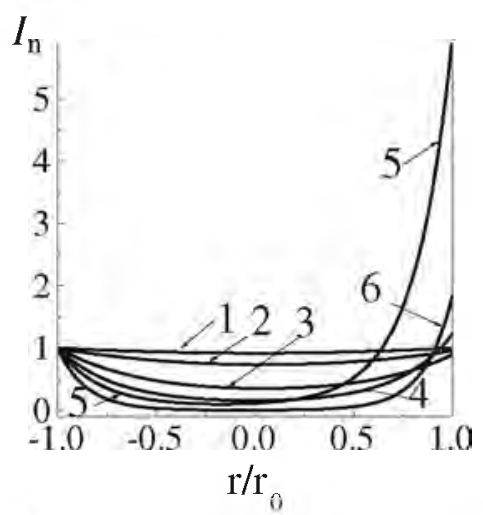

I

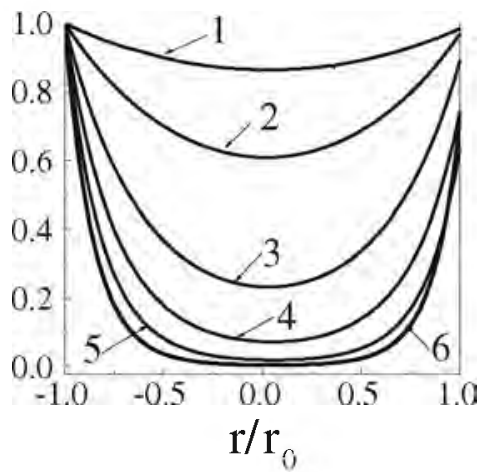

d

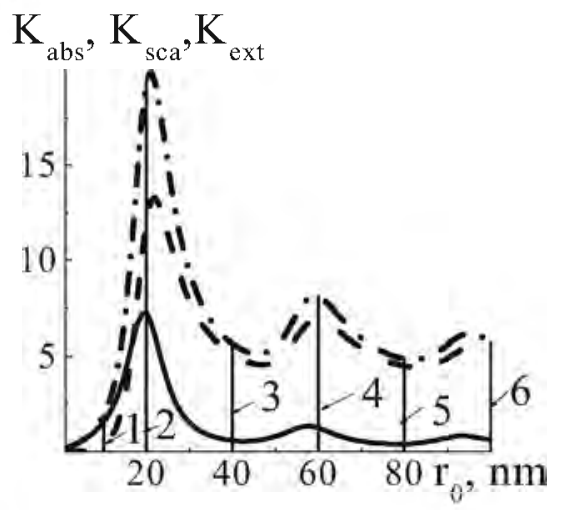

e

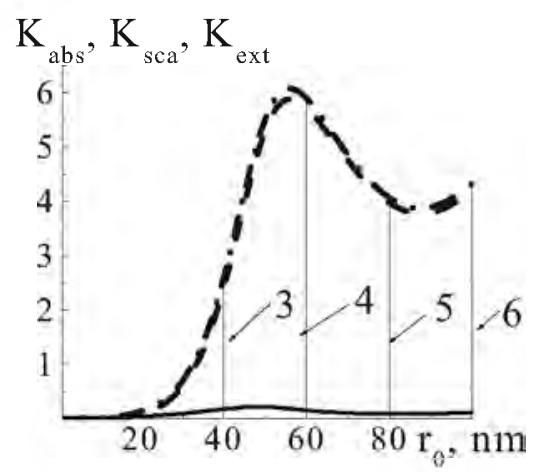

f

$\mathrm{K}_{\text {abs }}, \mathrm{K}_{\text {sea }}, \mathrm{K}_{\text {ext }}$

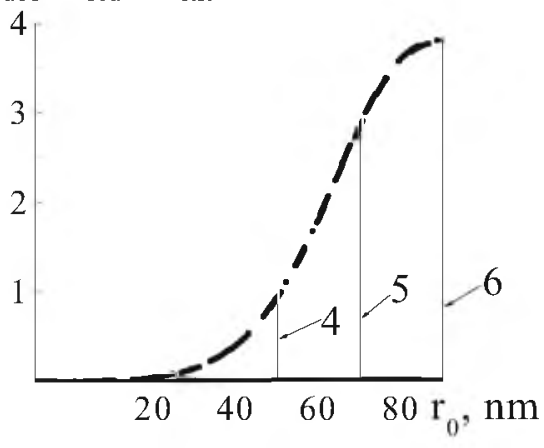

For the wavelength $\lambda=400 \mathrm{~nm}$, the relative intensity $I_{\mathrm{n}}$ has minimum in central part of NPs for NPs with all used radii instead of $r_{0}=40 \mathrm{~nm}$. The intensity $I_{\mathrm{n}}$ sharply increases at shadow part of NPs for $r_{0}=40$ and $80 \mathrm{~nm}$ and achieves the values up to 10-20 times greater in comparison with ones at irradiated side of NP. The factors $K_{\mathrm{abs}}, K_{\mathrm{sca}}$, and $K_{\text {ext }}$ undergo strong oscillations in the dependence on $r_{0}$. We can note that the minimal values of $K_{\text {abs }}, K_{\text {sca }}$, and $K_{\text {ext }}$ are placed at the values of about $r_{0} \sim 40$ and $80 \mathrm{~nm}$, and it is coordinated with maximal increase of $I_{\mathrm{n}}$ at shadow part of NPs for $r_{0} \sim 40$ and
$80 \mathrm{~nm}$ (Fig. 2d). It means that the extreme nanoscale increasing of $I_{n}$ in the shadow hemisphere of nanoparticles is determined by plasmon resonance effect in Ag NPs.

Increase of intensity $I_{\mathrm{n}}$ at shadow part of Ag NPs we can see for radius $r_{0}>60 \mathrm{~nm}$ and for wavelength $\lambda=532 \mathrm{~nm}$. The value of $I_{\mathrm{n}}$ is equal $I_{\mathrm{n}}\left(r / r_{0}=1\right) \sim 6$ for $r_{0}=80 \mathrm{~nm}$. The efficiency factors $K_{\text {sca, }}$ and $K_{\text {ext }}$ are much bigger than the absorption factor $K_{\text {abs }}$ for the range $r_{0}=10-100 \mathrm{~nm}$. Factors $K_{\mathrm{sca}}$ and $K_{\mathrm{ext}}$ have only one minimum at $r_{0} \sim 80-85 \mathrm{~nm}$, and it is correlated with one maximum of $I_{\mathrm{n}}\left(r / r_{0}=1\right)$ for $r_{0} \approx 80 \mathrm{~nm}$. 
Fig. 3 Spatial distributions of relative laser intensity $I_{\mathrm{n}}$ (arbitrary units) of laser radiation with wavelength $\lambda=532 \mathrm{~nm}$ inside spherical gold NPs $(\mathbf{a}, \mathbf{b}, \mathbf{c})$ and with the wavelength $\lambda=400 \mathrm{~nm}$ inside Ag NP (d, e, f) with $r_{0}=10$ $(\mathbf{a}, \mathbf{d}), 60(\mathbf{b}, \mathbf{e})$, and $100(\mathbf{c}, \mathbf{f}) \mathrm{nm}$. The arrows show the direction of laser radiation propagation
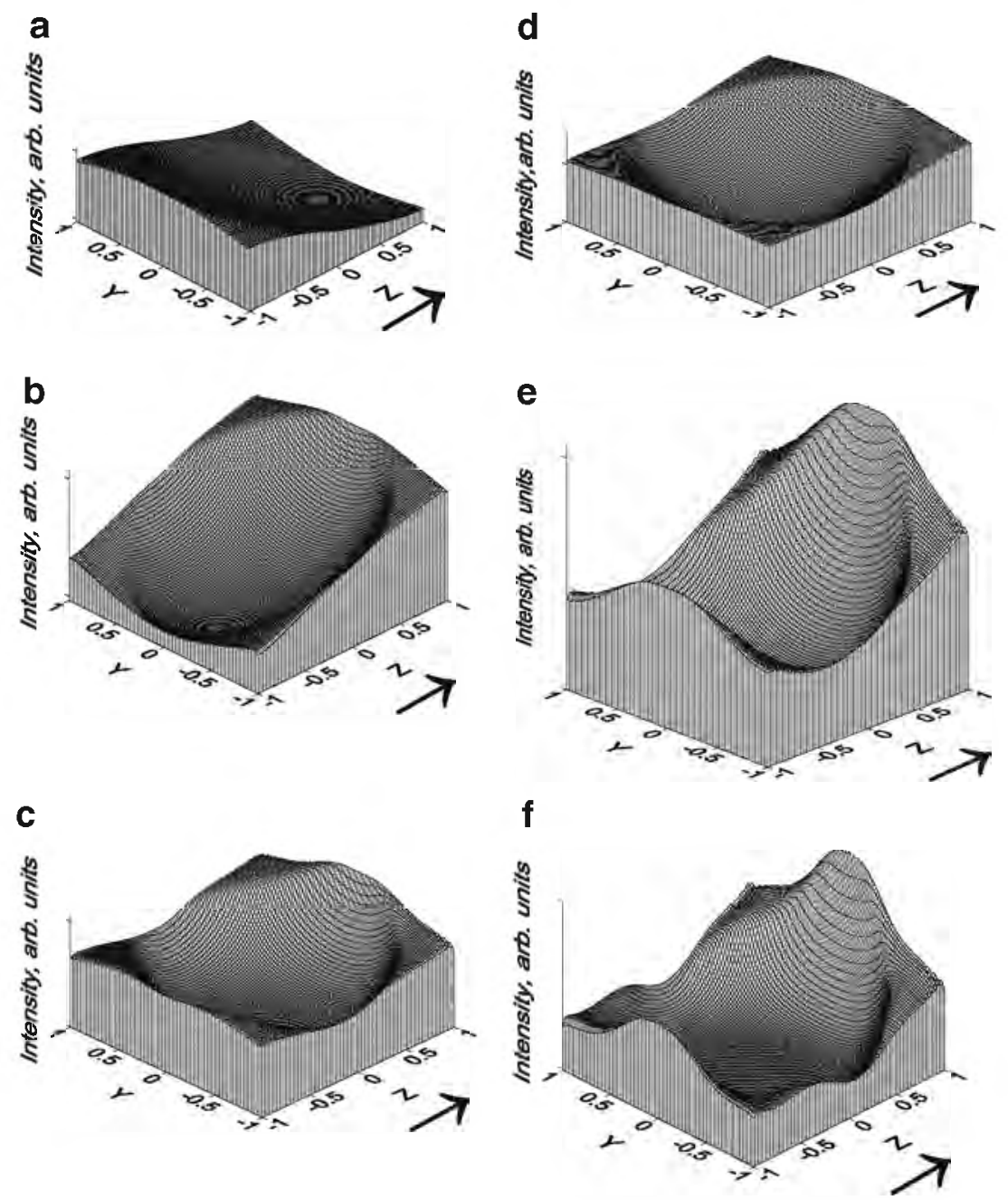

The intensity $I_{\mathrm{n}}$ decreases from irradiated surface to center of NP for infrared wavelength $800 \mathrm{~nm}$ with minimal value of $I_{\mathrm{n}}$ at the center of NP. $I_{\mathrm{n}}$ increases after that up to relative value $I_{\mathrm{n}}\left(r / r_{0}=1\right) \sim 0.6-0.98$. The values of $K_{\text {sca }}, K_{\text {ext }}>>K_{\text {abs }}$ and increase with increasing of $r_{0}$ in the range $r_{0}=10-100 \mathrm{~nm}$.

The relative intensity distributions inside gold and silver NPs for the wavelengths $\lambda=400,532$, and $800 \mathrm{~nm}$ have some general and particular features (peculiarities). The intensity $I_{\mathrm{n}}$ has maximal values at irradiated and shadow surfaces of NPs, and minimal values in central part for $r_{0}>20 \mathrm{~nm}$. The distribution of $I_{\mathrm{n}}$ is approximately homogeneous inside gold and silver NPs for small radiuses $r_{0}=10 \mathrm{~nm}$ and for all values of laser wavelengths mentioned above. Sharp increase of $I_{\mathrm{n}}$ in shadow part of $\mathrm{Au}\left(r_{0}=50-75 \mathrm{~nm}, \lambda=532 \mathrm{~nm}\right)$ and $\mathrm{Ag}\left(r_{0}=\right.$ $\left.30-55 \mathrm{~nm}, \lambda=400 \mathrm{~nm} ; r_{0}=70-95 \mathrm{~nm}, \lambda=532 \mathrm{~nm}\right)$ NPs is correlated with minimal values of $K_{\text {abs }}, K_{\text {sca, }}$, and $K_{\text {ext }}$. Such distributions of the intensity in NPs may be explained by the plasmonic properties of the Au and Ag NPs [1, 2, 27].

Figure 3 presents the spatial distributions of internal intensity $I_{\mathrm{n}}$ of laser radiation with wavelength $\lambda=532 \mathrm{~nm}$ inside spherical gold NPs (Fig. 3a-c) and the spatial distributions of internal intensity $I_{\mathrm{n}}$ of laser radiation with $\lambda=400 \mathrm{~nm}$ inside Ag NPs (Fig. 3d-f) with $r_{0}=10$ (a, d), 60 (b, e), and 100 (c, f) $\mathrm{nm}$. These pictures describe the spatial distributions of laser intensity inside overall volume of NPs and demonstrate the features of deformation of internal fields of laser intensity inside gold and silver NP with different sizes. The formation of emphasized minimal values of $K_{\mathrm{abs}}^{\mathrm{min}}, K_{\mathrm{sca}}^{\min }, K_{\mathrm{ext}}^{\mathrm{min}}$ for Au NPs, $\lambda=532 \mathrm{~nm}$ and for Ag NPs, $\lambda=400$ and $532 \mathrm{~nm}$ leads to the extreme light concentration of optical intensity determined by plasmonic resonance effect of radiation on NPs. General feature of light concentration in gold and silver NPs consist in the formation of approximately symmetric distribution $I_{n}$ with increasing of $I_{\mathrm{n}}$ in irradiated and shadow hemispheres of NPs and minimal value of $I_{\mathrm{n}}$ in the central part of NPs.

\section{Summary}

We have investigated the distributions of laser radiation intensities concentrated inside spherical gold and silver NPs with the radii in the range $10-100 \mathrm{~nm}$ under the action of laser 
irradiation with wavelengths $\lambda=400,532$, and $800 \mathrm{~nm}$. Main difference of our article from other articles consists in the investigation of internal radiation intensity distribution inside plasmonic NPs in comparison with the investigation of external near- or far field intensity distributions [1-11].

There are four characteristic distributions of laser intensity $I_{\mathrm{n}}$ inside gold and silver NPs for wavelengths 400,532 , and $800 \mathrm{~nm}$ :

(a) Approximately homogeneous distribution of relative intensity $I_{n}$ inside NP gold and silver NPs is realized for radiuses $r_{0} \leq 20 \mathrm{~nm}$ for all wavelengths mentioned above with accuracy of about 20-30\% (see Figs. 1 and 2).

(b) Maximal value of intensity $I_{n}$ lies at irradiated surface of gold NP is realized for wavelength $400 \mathrm{~nm}$ (see Fig. 1a)

(c) Maximal value of intensity $I_{\mathrm{n}}$ lies at shadow surface of $\mathrm{NP}$, and it is realized for gold NPs, radiation wavelength $532 \mathrm{~nm}, r_{0}=40-100 \mathrm{~nm}$ (see Fig. 1b), and for silver NPs, wavelength $400 \mathrm{~nm}\left(r_{0}=40-100 \mathrm{~nm}\right), 532 \mathrm{~nm}\left(r_{0}=80\right.$ $100 \mathrm{~nm}$ ) (see Fig. 2a, b).

(d) Approximately all maximal values of intensity $I_{n}$ lie simultaneously at irradiated and shadow surfaces of gold and silver NPs for wavelength $800 \mathrm{~nm}$, and it is realized for selected values of radii in the range $20 \div 100 \mathrm{~nm}$ (see Figs. $1 \mathrm{c}$ and $2 \mathrm{c}$ ).

The minimal values of the intensity $I_{\mathrm{n}}$ lie approximately in the central part of gold and silver NPs in all cases mentioned above.

In general, the realized situations in the distributions of the radiation intensity inside gold and silver NPS are determined by combined action of diffraction of radiation on spherical NP, extinction (absorption, scattering) of radiation inside material of NP, surface plasmon resonance effect and influence of NP radius $r_{0}$ and optical indexes of refraction and absorption of NP metal for concrete radiation wavelength. In some special (selected) cases, one or more mentioned processes are dominated under others and resulted intensity distribution $I_{n}$ is presented in Figs. 1, 2, and 3.

The nonhomogeneous intensity distributions inside NPs can lead to very interesting consequences. First of all, it can be nonhomogeneous heating of NP volume by short laser pulses. The nonhomogeneous melting of surface layer of NP can be realized after achievement of the melting temperature of NP material with reshaping of NP and cold NP nonmelting central part (case d) [14-16]. The nonhomogeneous surface evaporation of NP with possible formation of nano networks can be realized after achievement of evaporation temperature (case b, c) $[13,17]$. Increasing of laser intensity may lead to realization of optical breakdown and fragmentation of NP [19] taking into account the nonhomogeneous intensity distributions inside NPs. These possibilities can be used for special aims in selective laser nanotechnologies.
Here, we show new effect of extreme light intensity concentration in shadow semisphere of gold and silver NPs with localizing light at the nanoscale for selected values of NP sizes and radiation wavelengths. These results can be applied in nanophotonics for construction of new plasmonic antennas and photonic components, and for the laser technologies of the preparation and the treatment of NPs with definite sizes, shapes, structure, size distribution, etc.

The change of different materials (metals) and sizes of NPs, radiation wavelengths can realize different situations mentioned above for different applications in nanophotonics and laser nanotechnology. The laser intensity distributions and energy depositions inside NPs can essentially determine experimental results of laser action. This knowledge is of demand in such possible fields of applications as laser technology of NPs and nanophotonics.

\section{References}

1. Kreibig U, Vollmer M (1995) Optical properties of metal clusters. Springer, Berlin

2. Bohren CF, Huffman DR (1983) Absorption and scattering of light by small particles. Wiley, New York

3. Maier S (2007) Plasmonics: fundamentals and applications Springer, New York

4. Stockman M (2011) Nanoplasmonics: past, present and glimpse into future. Opt Express 19:22029-22106

5. Sonnefraud Y, Koh A, McComb D, Maier S (2012) Nanoplasmonics: engineering and observation of localized plasmon modes. Laser Photonics Rev 6:277-295

6. Zheludev N (2006) Single NP as photonic switch and optical memory element. J Optics A Pure Appl Opt 8:S1-S7

7. Schuller J et al (2010) Plasmonics for extreme light concentration and manipulation. Nat Mater 9:193-204

8. Colombo C, Krogstrup P, Nygård J, Brongersma ML, Fontcuberta A (2011) Engineering light absorption in single-nanowire solar cells with metal NPs. New J Phys 13:123026

9. Kauranen M, Zayats A (2012) Nonlinear plasmonics. Nat Photon 6: 737-748

10. Arnold M, Blaber M, Ford M (2014) Local plasmon resonances of metal-in-metal core-shells. Opt Express 22:3186-3198

11. Demichel O et al (2014) Selective excitation of bright and dark plasmonic resonances of single gold nanorods. Opt Express 22: $15088-15096$

12. Pustovalov VK (2005) Theoretical study of heating of spherical NPs in media by short laser pulses. Chem Phys 308:103-108

13. Werner D, Furube A, Okamoto T, Hashimoto S (2011) Femtosecond laser-induced size reduction of aqueous gold NPs: in situ and pump-probe spectroscopy investigations revealing coulomb explosion. J Phys Chem C 115:8503-8512

14. Fan C et al (2012) Gold NPs reshaped by ultrafast laser irradiation inside a silica-based glass, studied through optical properties. J Phys Chem C 116:2647-2655

15. Wang J et al (2011) Photothermal reshaping of gold NPs in a plasmonic absorber. Opt Express 19:14726-14734

16. Bruzzone S, Malvaldi M (2009) Local field effects on laser-induced heating of metal NPs. J Phys Chem C 113:15805-15810 
17. Pustovalov V (2011) Modeling of the processes of lasernanoparticle interaction taking into account temperature dependences of parameters. Laser Phys 21:906-912

18. Garwe F et al (2008) Optically controlled thermal management on the nanometer length scale. Nanotechnology 19: 055207

19. Giammanco F, Giorgetti E, Marsili P, Giusti A (2010) Experimental and theoretical analysis of photofragmentation of $\mathrm{Au}$ NPs by picosecond laser radiation. J Phys Chem C 114:3354-3363

20. Pustovalov VK, Smetannikov AS (2014) Analytical and computer modeling of thermal processes of laser interaction with single nanoparticle. RSC Adv 4:55760-55772

21. Astafyeva LG, Babenko VA (2004) Interaction of electromagnetic radiation with silicate spheroidal aerosol particles. J Quant Spectrosc Radiat Transf 88:9-14
22. Astafyeva L, Ledneva $H$ (2006) Thermal effect of radiation on dyedoped polysterene particle covered with deposited silver layer. Appl Opt 45:3878-3884

23. Li C, Cattawar GW, Zhai P-W (2005) Electric and magnetic energy density distributions inside and outside dielectric particles illuminated by a plane electromagnetic wave. Opt Express 13:4554-4559

24. Thormalen I, Straub J, Grigull U (1985) Refractive index of water and its dependence on wavelength, temperature and density. J Phys Chem Ref Data 14:933-945

25. Johnson PB, Christy RW (1972) Optical constants of the noble metals. Phys Rev B 6:4370-4379

26. Babenko VA, Astafyeva LG, Kuzmin VN (2003) Electromagnetic scattering in disperse media. Springer-Praxis, Berlin

27. Pustovalov VK, Babenko VA (2004) Optical properties of gold NPs at laser radiation wavelengths for laser applications in nanotechnology and medicine. Las Phys Lett 1:516-520 